\title{
Academic Skills in High-Functioning and Low-Functioning Chinese Children with Autism
}

\author{
Thomas L. Layton ${ }^{1}$ and Grace Hao,"* \\ ${ }^{1}$ Talk and Total Communication, Durham, NC 27713, USA \\ ${ }^{2}$ North Carolina Central University, Durham, NC 27707, USA
}

\begin{abstract}
Early academic skills among Chinese speaking children with ASD were compared to age matched peers who were typically developing and with intellectual disabilities. In general, children with ASD do perform less well on reading comprehension, writing, and math skills. The purpose of the current study was to gather preliminary data on the early academic skills of Chinese children with ASD. The findings indicated that HFA and LFA had less difficulty in acquiring beginning basic-academic skills, but had more difficulty in acquiring advanced academic skills compared to their peers. Yet, the two groups of Chinese students with HFA and LFA performed remarkably alike across the academic skills.
\end{abstract}

Keywords: Autism Spectrum Disorders, literacy, mathematical skills, Chinese.

\section{INTRODUCTION}

The early academic domains of reading, writing, and arithmetic abilities in Chinese children have not been well reported in the literature in spite of the fact that a growing number of individuals with ASD are being diagnosed [1-2]. The purpose of the current investigation was to gather preliminary data of early academic skills in Chinese children with ASD. A comparison of early reading, writing, and math skills was completed on: 1) a group of Chinese children with high-functioning autism (HFA) and a group of agematched peers who were typically developing (TD), and 2) a group of Chinese children with low-functioning autism (LFA) and a group of age-matched peers who were intellectually disabled (ID).

\section{Autism and Academics}

According to Jones, Happé, Golden et al. [3], students with Autism Spectrum Disorders (ASD) [4] often demonstrate difficulties in the classroom environment. Difficulties can vary from poor reading skill and poor writing skills, to inabilities in math skills such as completing problem-solving tasks. Yet, some students with ASD have been found to show good sight-word reading [5-8] are able to complete simple math calculations [1], and write basic letters [9]. Unfortunately, nearly all of these investigations have included only HFA students, although nearly half of ASD children are reported to be LFA [10]. Previous investigations have also been limited primarily to English speaking individuals while few studies have

*Address correspondence to this author at the North Carolina Central University, 710 Cecil Street Durham, NC 27707, USA; Tel: 919-530-7836; Fax: 919-484-0081; E-mail: jhao@nccu.edu included non-English speakers. Comparisons have also been reported with cognitive matched peers and not with age-matched children. Therefore, additional research is needed on the academic skills for both HFA and LFA children who are non-English speakers, such as Chinese children with their age-matched peers.

\section{Reading}

Reading requires being able to identify words (i.e., sight word reading or decoding real and nonsensewords) and being able to assemble words into meaning [11]. The ability to identify or decode sight words among children with HFA has indicated that phonologic, orthographic, and word identification skills are either acquired at a slower rate [5] or fall within the average range of abilities [7, 12]. However, a study by Nation et al. [7] found a wide variety of performances across three groups of individuals with ASD (i.e., HFA, atypical autism, Asperger's syndrome). Some of their children, for instance, read sight words accurately, but had poor reading comprehension skills; while some performed poorly on both real- and nonsense-words; some could read both successfully; and some were unable to read nonsense-words, but could read real words successfully. In essence, reading ability varied across the groups, with reading skills not being limited to any one particular group.

Reading comprehension, for the most part, has consistently been reported to be an area of difficulty for most children with ASD [12-13]. Cronin [12], for example, examined the relationship between oral language, decoding, and reading comprehension in children with ASD. Subjects, in the study, were administered a series of language and reading subtests from several standardized instruments. Cronin's findings indicated no relationship between phonological 
skills and decoding skills; however, there was a significant relationship between semantics and decoding skills, as well as between semantics and reading comprehension, and also between syntactic skills and reading comprehension. Ricketts et al. [13] found a difference in their adolescents with ASD on being able to complete word recognition and reading comprehension tasks. On a word recognition task only 45 percent of their subjects scored greater than one percentile below the mean, whereas nearly 60 percent of their subjects scored greater than one percentile below the mean on a reading comprehension task.

These findings, along with others, suggest a variation in reading skills in English speaking children with HFA as well as different levels of reading accuracy. Generally speaking, children with HFA tend to perform better on decoding sight words, but less well on nonsense-words and on reading comprehension skills.

\section{Math}

In the area of mathematics, individuals with HFA tend to have difficulty in most areas, but especially in the area of math problem-solving skills [14]. A review of 18 articles by Chiang and Lin [1] found students with HFA generally had average mathematical abilities in simple calculation (see specifically 9,15 ]: other studies have reported that students with HFA display learning disabilities in math skills [16-17]. Most students, however, with ASD are reported to be successful in math counting, simple calculation, but not math problem-solving skills.

More research obviously is needed in the area of math in order to obtain a clearer understanding of math abilities among students with ASD, particularly in the areas of math facts, calculation, and problem solving. In addition, research is lacking among children with LFA and among Chinese children with ASD.

\section{Writing}

Most research investigations in writing skills have reported deficiencies among students with ASD [1719]. For instance, Mayes and Calhoun [17] found approximately 60 percent of their 164 HFA students had writing learning disabilities. The areas of difficulty, for a large number of the students, were with story composition, lower scores in story construction, and grammatical aspects of story constructions.

Other findings, indicate HFA students consistently demonstrate weaknesses in written expression and graphomotor skills [20]. According to Schaefer Whitby and Manci [20], causes for the graphomotor weaknesses could be due to poor motor coordination, whereas written expression could be due to the inability to organize ideas or an inability to attend. Troyb et al. [9] also suggested that some students with HFA demonstrate good writing skills, such as the arbitrary conventions of written language, language use in writing, as well as the ability to construct a story. Furthermore, Troyb et al. found that written narratives were similar in length and contained a number of comparable descriptions of social, emotional, cognitive, and causal processes compared to their peers.

Writing skills, therefore, appear to be an area of weakness for some students with ASD, but many are able to formulate letters, write sentences, and develop clear ideas. How Chinese children with ASD perform on such writing tasks, unfortunately, are unknown and needs further investigation.

\section{Present Study}

Reading, writing, and math skills among children with ASD are important for their success in school and for improving their social lifestyle in the community. In general, children with ASD typically do less well on reading comprehension, writing skills, and math problem solving tasks, but do better on reading sight words and simple math calculations. It is unclear whether such abilities also occur among children who are LFA or among Chinese students with ASD. Therefore, the purpose of the current study was to gather preliminary data on the academic skills of Chinese children with ASD. First, we compared the academic skills between a group of Chinese students with HFA and a group of age-matched peers who were typically developing (TD). Second, we compared the academic skills between a group of Chinese students with LFA and a group of age-matched peers who were intellectually disabled (ID). Third, we explored the academic skills between the groups of HFA and LFA students for any similar or different patterns of performance. The academic areas assessed were early reading skills, writing skills, and math skills.

\section{MATERIALS AND METHODS}

\section{Participants and Setting}

A total of 273 children from China were included in this study. Of that number 35 were females and 238 were males. All of the participants spoke Mandarin as their primary language. The children were recruited 
from nine different cities of Eastern China. There were 98 children diagnosed as HFA (mean age 60.57 months), 103 children diagnosed as LFA (mean age 57.18 months), 32 children diagnosed as TD (mean age 49.58 months), and 40 children diagnosed as ID non-autism (mean age 57.65 months). The LFA group consisted of children who were previously labeled as Classic Autism or Low Functioning Autism. An interdisciplinary team reviewed the results of several standard diagnostic tests in order to come up with a consensus diagnosis of LFA, HFA, and ID based on Diagnostic and Statistical Manual-IV [21], the ICD-10 Classification of Mental and Behavioral Disorders criteria [22] and the Chinese Classification of Mental Disorders and Diagnostic Criteria-3rd Edition (CCMD3) [23].

The group of children labeled TD was recruited from a population that received routine physical checkups at the Pediatric Unit of the hospital. The children identified as ID were from the same hospitals but were previously diagnosed with a history of intellectual disabilities. The ID children were administered the WISC-Hong Kong [24] test and had scored less than 70 points in order to qualify for the current study.

\section{Participant's Age Groups}

Attempts were made to include children across four age groups to assure representative samples among toddlers, preschoolers, kindergarteners, and early elementary school age children. The four age groups were: $24-35$ months $(n=36), 36-47$ months $(n=57), 48$ 59 months $(n=69)$, and 60-96 months $(n=112)$. Table 1 shows the mean ages across the four groups as well as their level of statistical significance. As can be seen, no significant differences occurred between all of the ages for the matched groups. Thus, this data indicates a good match between each ASD age group and the controls

\section{Inclusionary Criteria}

All of the children were included in the study based on a clinical diagnosis of ASD or non-ASD as determined by an interdisciplinary team of trained Chinese developmental pediatricians and developmental psychologists, who routinely evaluate children with autism and children with developmental disabilities. A regimen of clinical diagnostic procedures included: an evaluation by either the $A D I-R$ [25] or the Social Responsiveness Scale (SRS) [26], along with a thirty-minute clinical observation-protocol. The clinical observation-protocol included child observations and parent questionnaires, in the areas of motor and stereotyped behaviors, social interaction, play skills, and language skills. All of the children received a diagnosis and grouping subtypes based on the Diagnostic and Statistical Manual-IV [21] and The Chinese Classification of Mental Disorders and Diagnostic Criteria- ${ }^{\text {rd }}$ Edition [23].

Inclusionary criteria, for the typical developing control subjects, included children with average IQ who presented without any developmental disabilities or speech and language disorders. Inclusionary criteria for the subjects with ID included children who had been previously diagnosed with intellectual disabilities by a developmental pediatrician.

\section{Measures}

There are no standardized tests in China currently available to measure academic performance for

Table 1: Descriptive Statistics for Age Differences between Groups

\begin{tabular}{|c|c|c|c|c|c|c|c|c|c|}
\hline \multicolumn{5}{|c|}{ Group HFA* TD* } & \multicolumn{5}{|c|}{ Group LFA* ID* } \\
\hline $\begin{array}{c}\text { Age } \\
\text { groups }\end{array}$ & $\begin{array}{l}\text { HFA Means } \\
\text { (sd) }\end{array}$ & $\begin{array}{l}\text { TD Means } \\
\text { (sd) }\end{array}$ & $F$ value & $p$-value & $\begin{array}{c}\text { Age } \\
\text { groups }\end{array}$ & $\begin{array}{l}\text { LFA Means } \\
\text { (sd) }\end{array}$ & $\begin{array}{l}\text { ID Means } \\
\text { (sd) }\end{array}$ & $F$ value & $p$-value \\
\hline$(n=16)$ & $(2.47)$ & $(2.37)$ & & & $(n=20)$ & $(2.93)$ & $(4.12)$ & & \\
\hline $36-47$ & 43.74 & 40.78 & 3.59 & .069 & $36-47$ & 44.83 & 46.00 & .146 & .705 \\
\hline $48-59$ & 52.55 & 55.40 & 4.67 & .060 & $48-59$ & 54.88 & 53.67 & .850 & .363 \\
\hline$(n=32)$ & $(2.37)$ & $(2.91)$ & & & $(n=36)$ & $(3.78)$ & $(3.55)$ & & \\
\hline $60+$ & 76.29 & 70.33 & 1.69 & .198 & $60+$ & 75.02 & 72.41 & .886 & .351 \\
\hline$(n=54)$ & $(10.85)$ & $(7.17)$ & & & $(n=58)$ & $(9.54)$ & $(9.84)$ & & \\
\hline
\end{tabular}

HFA = high functioning autism spectrum disorders; TD= typically developing; LFA = low functioning autism spectrum disorders; ID = intellectually disabled. 
children at the ages selected in this study. Thus, a nonstandardized testing protocol was developed to collect data on the early academic skills from the three domains: reading, writing, and math. There were 14 skills assessed in the protocol. The 14 skills were divided across three areas: 1) Reading (four items), 2) Writing (four items), and 3) Mathematics (six items). Scoring for each item consisted of assigning a score of 0 - if the child had mastered the skill being tested, a score of 1 - if the child had developed emerging skills on the item being tested, and a score of 2- if the child had not clearly demonstrated the development of the item being tested.

\section{Procedures}

Each child was administered the academic protocol by a trained Chinese professional. A parent or caregiver had been always present to assist in the assessment. Testing was completed in a single setting. Breaks were provided when necessary. The total assessment time for completing the protocol was approximately 20 minutes. The correct response of each item was provided by the examiner, but the scoring system was not shared. The test examiners, therefore, did not know the total scores for each child being assessed.

\section{Statistical Analysis}

The results were analyzed using the SPSS-21 program [27]. Group comparisons were completed initially between the individuals who were HFA and the children with TD. Again, the same comparisons were made between the children with LFA and their peers who were ID. We subsequently conducted an exploratory factor analysis (EFA) following an initial inter-item correlation analysis with a principal factor
Varimax rotation. This was performed between the HFA children and the TD children, as well as between the LFA children and the ID children. To determine factor loadings we followed the recommendation of Kaiser [28] by using eigenvalues greater than 1. To determine which variables within a factor made up that factor, we used a conservative loading of 0.707 per variable, as recommended by Stevens [29].

\section{RESULTS}

\section{Academic Skills Across Groups}

All alpha scores were set at a .01 conservative level. A robust ANOCVA was analyzed in the group of children with HFA versus the group with TD, along with a covariate of age. A similar ANOCVA was analyzed in the group of children with LFA and the children with ID with a covariate of age. Both overall ANOCVAs were found to be non-statistically significant (HFA: $F=0.64$, $\mathrm{p}=.423, \mathrm{Eta}^{2}=.005 ;$ LFA: $\mathrm{F}=.372, \mathrm{p}=.372, \mathrm{Eta}^{2}=.003$ ); however, the covariates of age were found to be statistically significant and appeared to affect the outcomes (i.e., HFA: age covariate $F=134.77, p=.001$, Eta2 $=515$; LFA: age covariate $F=65.68, p=.001$, Eta2 $=.319$ ). A series of post-hoc mean comparisons (i.e., Tukey-B) were completed to determine which age groupings were statistically different (see Table 2). In both sets of comparisons, the children with ASD differed significantly from their control groups without ASD. This suggests the children with ASD performed less well on the academic skills than did their agematched peers.

\section{Factor Analysis HFA and TD}

Inter-correlational analyses followed by factor analyses were computed for the academic skills to

Table 2: Descriptive Statistics for Total Academic Scores Across Groups

\begin{tabular}{|c|c|c|c|c|c|c|c|c|c|}
\hline \multicolumn{5}{|c|}{ Group HFA* TD* } & \multicolumn{5}{|c|}{ Group LFA* ID* } \\
\hline $\begin{array}{l}\text { Age } \\
\text { groups }\end{array}$ & $\begin{array}{l}\text { HFA Means } \\
\text { (sd) }\end{array}$ & $\begin{array}{l}\text { TD Means } \\
\text { (sd) }\end{array}$ & $F$ value & $p$-value & $\begin{array}{c}\text { Age } \\
\text { groups }\end{array}$ & $\begin{array}{l}\text { LFA Means } \\
\text { (sd) }\end{array}$ & $\begin{array}{l}\text { ID Means } \\
\text { (sd) }\end{array}$ & $F$ value & $p$-value \\
\hline $24-35$ & 25.22 & 22.58 & 24.81 & .0001 & $24-35$ & 26.33 & 27.80 & 158.33 & .0001 \\
\hline$(n=16)$ & $(3.63)$ & (3.91) & & & $(n=20)$ & $(1.64)$ & $(0.45)$ & & \\
\hline $36-47$ & 23.00 & 21.44 & 40.21 & .0001 & $36-47$ & 24.96 & 23.00 & 285.30 & .0001 \\
\hline$(n=28)$ & $(5.47)$ & (3.54) & & & $(n=29)$ & (3.31) & $(7.04)$ & & \\
\hline $48-59$ & 19.05 & 18.70 & 45.66 & .0001 & $48-59$ & 21.95 & 21.50 & 214.71 & .0001 \\
\hline$(n=32)$ & $(6.88)$ & $(2.67)$ & & & $(n=36)$ & $(4.99)$ & $(4.40)$ & & \\
\hline $60+$ & 16.07 & 10.50 & 69.98 & .0001 & $60+$ & 16.98 & 20.88 & 400.72 & .0001 \\
\hline$(n=54)$ & (8.89) & (7.04) & & & $(n=58)$ & $(8.84)$ & $(5.82)$ & & \\
\hline
\end{tabular}

HFA = high functioning autism spectrum disorders; TD= typically developing; LFA = low functioning autism spectrum disorders; ID = intellectually disabled. 
Table 3: Correlation for Academics: Typical Developing (on Top) HFA (on Bottom)

\begin{tabular}{|c|c|c|c|c|c|c|c|c|c|c|c|c|c|c|}
\hline & A & B & C & D & $E$ & $F$ & G & $\mathbf{H}$ & I & $\mathbf{J}$ & K & L & M & $\mathbf{N}$ \\
\hline A & - & .665 & .340 & .421 & .345 & .405 & .315 & .053 & .286 & .350 & .519 & .588 & .296 & .509 \\
\hline B & .732 & - & .316 & .414 & .294 & .281 & .448 & .149 & .291 & .405 & .345 & .448 & .226 & .388 \\
\hline C & .653 & .617 & - & .598 & .763 & .498 & .149 & .053 & .286 & .350 & .462 & .588 & .296 & .509 \\
\hline D & .658 & .565 & .719 & - & .664 & .638 & .136 & .204 & .205 & .361 & .546 & .617 & .449 & .581 \\
\hline$E$ & .669 & .638 & .750 & .828 & - & .777 & .179 & .131 & .190 & .270 & .499 & .641 & .463 & .411 \\
\hline$F$ & .429 & .430 & .545 & .605 & .710 & - & .234 & .025 & .107 & .189 & .659 & .751 & .812 & .651 \\
\hline G & .627 & .561 & .577 & .630 & .610 & .446 & - & .409 & .299 & .347 & .249 & .468 & .236 & .406 \\
\hline $\mathrm{H}$ & .455 & .435 & .535 & .543 & .516 & .600 & .499 & - & .548 & .587 & .140 & .010 & .190 & .166 \\
\hline 1 & .645 & .651 & .516 & .498 & .460 & .345 & .426 & .360 & - & .724 & .148 & .126 & .064 & 109 \\
\hline $\mathrm{J}$ & .719 & .708 & .559 & .524 & .522 & .318 & .551 & .365 & .773 & - & .262 & .223 & .112 & 193 \\
\hline K & .517 & .474 & .646 & .647 & .689 & .728 & .506 & .650 & .384 & .337 & - & .734 & .459 & .788 \\
\hline L & .498 & .457 & .617 & .660 & .660 & .628 & .590 & .638 & .302 & .371 & .797 & - & .618 & .827 \\
\hline M & .346 & .351 & .488 & .528 & .521 & .549 & .419 & .651 & .220 & .303 & .677 & .650 & - & .653 \\
\hline $\mathrm{N}$ & .265 & .236 & .360 & .416 & .461 & .499 & .416 & .603 & .140 & .209 & .582 & .643 & .707 & - \\
\hline
\end{tabular}

$A=$ basic alphabet; $B=$ reads simple words; $C=$ writes name; $D=$ writes pinyin letters; $E=$ writes simple words; $F=$ writes short sentences to dictation; $G=$ print awareness; $\mathrm{H}=$ comprehends short paragraphs; $\mathrm{I}=$ counts to 20 ; $\mathrm{J}=$ identify numbers; $\mathrm{K}=$ adds one and two column figures; $\mathrm{L}=$ simple subtraction; $\mathrm{M}=$ counts by $2 \mathrm{~s}$, $3 \mathrm{~s}$, and $4 \mathrm{~s} ; \mathrm{N}=$ math problem solving.

determine their relative relationships. Table 3 contains the TD group's inter-correlations in the upper matrix and the HFA group in the lower matrix. As can be seen, few of the correlations exceeded the criteria by 0.707 . There were only eleven variables for the HFA group and eight for the TD group that exceeded criteria. This suggests a remarkable independence across the variables.

Table 4 shows the Varimax rotated sums of square for the HFA and the TD groups. The table indicates two factor loadings for the HFA and four loadings for the TD group. The two factors for the HFA group accounted for approximately 72 percent of the variance, whereas the four factors for the TD group accounted for approximately 80 percent of the variance.

Table 5 presents the rotated loadings for each of the factors for the HFA group. The first factor had four variables greater than 0.707 . These were: 1 ) basic alphabet (Variable A), 2) simple words (Variable B), 3) count to 20 (Variable I), and 4 identify numbers (Variable J). The second factor had six variables: 1 ) writes short sentences for dictation (Variable F), 2) comprehends information in short paragraphs (Variable $\mathrm{H})$, 3) adds one and two columns (Variable K), 4) understands simple subtraction (Variable L), 5) counts by $2 \mathrm{~s}, 3 \mathrm{~s}$, and $4 \mathrm{~s}$ (Variable $\mathrm{N}$ ), and 6 ) solves a simple math problem (Variable M).

The first factor clearly represents early developing or beginning basic-academic skills, such as knowing the alphabet, reading words, and knowing the numbers. The second factor can be considered to include later developing basic-academic skills, such as reading for comprehension and higher math skills.

Table 5 also presents the academic skills for the TD group distributed across their four factors. The first factor contains four variables: writes short sentences

Table 4: Rotated Sums of Square Loadings for HFA and Typical Developing (TD)

\begin{tabular}{|c|c|c|c|c|c|}
\hline \multirow{2}{*}{ Component } & \multicolumn{2}{|c|}{ HFA } & \multicolumn{2}{|c|}{ Component } & \multicolumn{2}{|c|}{ Total } & \% variance \\
\cline { 2 - 6 } & Total & \% variance & & 3.603 & 24.021 \\
\hline \hline 1 & 5.455 & 36.364 & 1 & 2.940 & 2.882 \\
\hline 2 & 5.393 & 35.956 & 2 & 2.557 & 19.599 \\
\hline
\end{tabular}


Table 5: Rotated Matrix for HFA and Typical Developing (TD)

\begin{tabular}{|c|c|c|c|c|c|c|c|}
\hline \multicolumn{4}{|c|}{ HFA } & \multicolumn{4}{|c|}{ TD } \\
\hline \multicolumn{4}{|c|}{ Components } & \multicolumn{4}{|c|}{ Components } \\
\hline Items & 1 & 2 & Items & 1 & 2 & 3 & 4 \\
\hline$A$ & .848 & .278 & $A$ & .205 & .787 & .298 & .043 \\
\hline B & .835 & .240 & B & .069 & .853 & .228 & .143 \\
\hline $\mathrm{C}$ & .642 & .514 & $\mathrm{C}$ & .271 & .123 & .769 & .198 \\
\hline $\mathrm{D}$ & .603 & .584 & $\mathrm{D}$ & .343 & .243 & .765 & --- \\
\hline$E$ & .601 & .613 & $E$ & .404 & .074 & .801 & .052 \\
\hline $\mathrm{F}$ & .304 & .732 & $\mathrm{~F}$ & .789 & .081 & .483 & .027 \\
\hline G & .618 & .458 & G & .355 & .578 & --- & .374 \\
\hline $\mathrm{H}$ & .310 & .742 & $\mathrm{H}$ & .216 & .058 & --- & .866 \\
\hline 1 & .829 & .067 & I & -- & .174 & .265 & .828 \\
\hline $\mathrm{J}$ & .871 & .105 & $\mathrm{~J}$ & --- & .268 & .331 & .812 \\
\hline $\mathrm{K}$ & .331 & .816 & $\mathrm{~K}$ & .655 & .313 & .350 & .059 \\
\hline L & .317 & .816 & L & .711 & .437 & .409 & --- \\
\hline M & .140 & .829 & M & .863 & .022 & .147 & .083 \\
\hline $\mathrm{N}$ & .022 & .825 & $\mathrm{~N}$ & .791 & .368 & .228 & .039 \\
\hline
\end{tabular}

$\mathrm{A}=$ basic alphabet; $\mathrm{B}=$ reads simple words; $\mathrm{C}=$ writes name; $\mathrm{D}=$ writes pinyin letters; $\mathrm{E}=$ writes simple words; $\mathrm{F}=$ writes short sentences to dictation; $\mathrm{G}=$ print awareness; $\mathrm{H}=$ comprehends short paragraphs; $\mathrm{I}=$ counts to $20 ; \mathrm{J}=$ identify numbers; $\mathrm{K}=$ adds one and two column figures; $\mathrm{L}=$ simple subtraction; $\mathrm{M}=\mathrm{counts}$ by $2 \mathrm{~s}$, $3 \mathrm{~s}$, and $4 \mathrm{~s} ; \mathrm{N}=$ math problem solving.

for dictation (Variable $\mathrm{F}$ ) and three higher math skills (i.e., L-subtraction, M-counts by $2 \mathrm{~s}, 3 \mathrm{~s}$, and $4 \mathrm{~s} ; \mathrm{N}$ solves math problems). Similarly, the second factor contains two variables: basic alphabet (Variable $\mathrm{C}$ ), and reads simple words (Variable $B$ ). The third factor contains three loadings: writes name (Variable $\mathrm{C}$ ), writes pinyin letters (Variable D), and writes simple words (Variable E). The fourth factor contains three loadings: comprehends information in short paragraphs (Variable $\mathrm{H}$ ), counts to 20 (Variable I), and identify numbers (Variable J).

Therefore, the first factor, for the TD group, represents later developing basic-academic skills while the second Factor represents the early developing beginning basic-academic skills. The third factor represents beginning writing-skills, and the fourth factor represents a beginning math-skills. It is unclear why variable $\mathrm{H}$ (i.e., comprehends information in short paragraphs) loaded on the fourth factor since it should more appropriately load on the first Factor.

\section{Factor Analysis LFA and ID}

As done previously, similar inter-correlation analyses and factor analyses were computed for the LFA and ID groups. Table 6 contains the intercorrelations with the ID group in the upper matrix and the LFA in the lower matrix. There were ten variables that exceeded the criteria of 0.707 for the LFA group and seven variables for the group ID. This again suggests independence within these variables.

Table 7 shows the Varimax rotated sums of square for the LFA and ID groups. The table indicates two factor loadings for the LFA group and four factor loadings for the ID group. The two factors for the LFA group accounted for nearly 70 percent of the variance, and the four factors for the ID group accounted for nearly 81 percent of the variance.

Table 8 presents the rotated loadings for each of the factors. The LFA first factor had five variables greater than 0.707. These were: 1) basic alphabet (Variable A), 2 read simple words (Variable B), 3) writes name (Variable C), 4) counts to 20 (Variable I), and 5) identify numbers (Variable $\mathrm{J}$ ). This factor represents knowledge and understanding of beginning basic-academic skills, similar to the first factor for the HFA group as mentioned previously. The second factor had six loadings: 1) writes short sentences for dictation (Variable F), 2) comprehends information in short paragraphs (Variable $\mathrm{H}$ ), 3) adds one and two column figures (Variable $\mathrm{KI}$ ), 4) understand simple subtraction (Variable N), 5) counts by 2s, 3s, and 4s (Variable M), and 6) solves simple math problems (Variable N). This 
Table 6: Correlation for Academics: Intellectually Disabled (on Top) LFA (on Bottom)

\begin{tabular}{|c|c|c|c|c|c|c|c|c|c|c|c|c|c|c|}
\hline & A & B & C & D & $E$ & $F$ & $\mathbf{G}$ & $\mathbf{H}$ & I & $\mathbf{J}$ & $K$ & L & M & $\mathbf{N}$ \\
\hline A & - & .824 & .688 & .645 & .554 & .247 & .626 & .316 & .432 & .732 & .412 & .479 & .333 & .247 \\
\hline B & .780 & - & .608 & .717 & .484 & .329 & .666 & .467 & .513 & .590 & .360 & .356 & .076 & .125 \\
\hline C & .707 & .607 & - & .716 & .523 & .342 & .350 & .232 & .268 & .574 & .571 & .414 & .480 & .342 \\
\hline D & .591 & .489 & .798 & - & .616 & .391 & .584 & .414 & .391 & .496 & .653 & .350 & .166 & .076 \\
\hline$E$ & .700 & .626 & .792 & .750 & - & .518 & .461 & .478 & .077 & .409 & .264 & .725 & .504 & .518 \\
\hline$F$ & .434 & .341 & .649 & .595 & .553 & - & .314 & .381 & .132 & .182 & .385 & .280 & .043 & .026 \\
\hline G & .520 & .576 & .543 & .455 & .496 & .405 & - & .539 & .489 & .522 & .163 & .339 & .072 & .119 \\
\hline $\mathrm{H}$ & .464 & .358 & .472 & .430 & .141 & .572 & .313 & - & .261 & .478 & .262 & .275 & .075 & .067 \\
\hline I & .643 & .690 & .508 & .407 & .468 & .358 & .464 & .317 & - & .635 & .220 & .093 & .106 & .259 \\
\hline $\mathrm{J}$ & .742 & .800 & .612 & .499 & .562 & .387 & .554 & .377 & .878 & - & .304 & .300 & .224 & .182 \\
\hline $\mathrm{K}$ & .528 & .433 & .671 & .522 & .712 & .579 & .418 & .513 & .369 & .440 & - & .175 & .107 & .043 \\
\hline L & .464 & .343 & .607 & .531 & .661 & .658 & .285 & .625 & .330 & .394 & .825 & - & .758 & .629 \\
\hline$M$ & .223 & .035 & .199 & .228 & .229 & .374 & .160 & .469 & .145 & .173 & .387 & .431 & - & .813 \\
\hline $\mathrm{N}$ & .260 & .112 & .348 & .388 & .391 & .608 & .299 & .684 & .169 & .202 & .569 & .631 & .635 & - \\
\hline
\end{tabular}

$\mathrm{A}=$ basic alphabet; $\mathrm{B}=$ reads simple words; $\mathrm{C}=$ writes name; $\mathrm{D}=$ writes pinyin letters; $\mathrm{E}=$ writes simple words; $\mathrm{F}=$ writes short sentences to dictation; $\mathrm{G}=$ print awareness; $\mathrm{H}=$ comprehends short paragraphs; $\mathrm{I}=$ counts to $20 ; \mathrm{J}=$ identify numbers; $\mathrm{K}=$ adds one and two column figures; $\mathrm{L}=$ simple subtraction; $\mathrm{M}=\mathrm{counts}$ by $2 \mathrm{~s}$ $3 \mathrm{~s}$, and $4 \mathrm{~s} ; \mathrm{N}=$ math problem solving.

second factor, therefore, represents knowledge of later developing basic-academic skills and understanding of math skills.

The ID group had four factor loadings. In the first factor, there were five variables that loaded: 1) basic alphabet, 2) read simple words, 3) counts to 20,4) identify numbers, and 5) print awareness concepts. (The variable "writes pinyin letters" did not load on this factor). Thus, the first factor, with the exception of writing pinyin, can be considered to represent knowledge of beginning basic-academic skills similar to the LFA group. The ID second factor had only three loadings and each were in the area of math (i.e., understands simple subtraction; counts by $2 \mathrm{~s}, 3 \mathrm{~s}$, and $4 \mathrm{~s}$; and solves simple math problems). Thus, the second factor represents knowledge of math skills. The third factor had a single loading: adds one and two columns, whereas the fourth factor also had only a single loading: writes simple sentences to dictation.
This last variable requires writing, memory, and higher academic knowledge.

\section{Comparison between HFA and LFA}

We compared just the two groups of children with ASD for any similarities and differences across the academic skills. One common aspect between the two groups with ASD was that both groups had only two factor loadings across the academic skills, whereas the TD and ID control groups had four loadings. In addition, the first factor for the two groups with ASD contained similar variable-loadings (i.e., basic alphabet, reading simple words, counting to 20 , identifying numbers). This factor was considered to represent early academic skills. Their second factor loading contained mostly math skills (i.e., adding one and two column figures; understanding simple subtraction; counting by $2 \mathrm{~s}, 3 \mathrm{~s}$, and $4 \mathrm{~s}$; and solving simple math problems). Three academic skills did not load on either the first or second

Table 7: Rotated Sums of Square Loadings for LFA and Intellectually Disabled (ID)

\begin{tabular}{|c|c|c|c|c|c|}
\hline \multicolumn{2}{|c|}{ LFA } & \multicolumn{2}{c|}{ ID } \\
\hline Component & Total & \% variance & Component & Total & 4.513 \\
\hline \hline 1 & 6.056 & 40.375 & 1 & 3.179 & 30.085 \\
\hline 2 & 4.442 & 29.614 & 2 & 2.230 & 192 \\
\hline & & & 3 & 2.198 & 14.652 \\
\hline
\end{tabular}


Table 8: Rotated Matrix for LFA and Intellectually Disabled (ID)

\begin{tabular}{|c|c|c|c|c|c|c|c|}
\hline \multicolumn{4}{|c|}{ LFA } & \multicolumn{4}{|c|}{ ID } \\
\hline Items & 1 & 2 & Items & 1 & 2 & 3 & 4 \\
\hline A & .844 & .265 & $A$ & .773 & .344 & .352 & .109 \\
\hline B & .914 & --- & B & .785 & --- & .317 & .304 \\
\hline$E$ & .677 & .514 & $E$ & .255 & --- & .223 & .612 \\
\hline$F$ & .355 & .719 & $\mathrm{~F}$ & --- & --- & .394 & .761 \\
\hline G & .678 & .211 & G & .750 & --- & --- & .475 \\
\hline $\mathrm{H}$ & .296 & .732 & $\mathrm{H}$ & .413 & --- & --- & .691 \\
\hline $\mathrm{K}$ & .429 & .711 & $\mathrm{~K}$ & .137 & --- & .885 & .146 \\
\hline$L$ & .316 & .808 & $L$ & .221 & .799 & --- & .336 \\
\hline M & --- & .706 & M & --- & .927 & --- & --- \\
\hline $\mathrm{N}$ & --- & .879 & $\mathrm{~N}$ & .111 & .944 & --- & --- \\
\hline
\end{tabular}

$A=$ basic alphabet; $B=$ reads simple words; $C=$ writes name; $D=$ write pinyin letters; $E=$ writes simple words; $F=$ writes short sentences to dictation; $G=$ print awareness; $\mathrm{H}=$ comprehends short paragraphs; $\mathrm{I}=$ counts to $20 ; \mathrm{J}=$ identify numbers; $\mathrm{K}=$ adds one and two column figures; $\mathrm{L}=$ simple subtraction; $\mathrm{M}=$ counts by $2 \mathrm{~s}$, $3 \mathrm{~s}$, and $4 \mathrm{~s} ; \mathrm{N}=$ math problem solving.

factor. These were: writing pinyin letters, writing simple words, and print awareness concepts. Therefore, it can be concluded that the two groups of children with ASD, regardless of their levels of autism, performed remarkably alike across the academic skills assessed; but, both groups presented limited writing skills, less reading comprehension skills, as well as problems in math problem-solving skills.

\section{DISCUSSION}

\section{Current Finding and Previous Research}

The current findings of poorer reading skills in Chinese children with HFA support previous research that also demonstrates a difference in reading skills [5, $7]$, but not reading accuracy [6, 8]. The current Chinese children performed better in basic reading skills, such as knowing the alphabet and reading simple word reading; but, they performed less well on more advanced academic skills, such as reading for comprehension. The current investigation extends previous findings by including children who were LFA. Thus, taken together, the current findings and most previous investigations support the fact that children with ASD, across different levels of functioning, have less difficulty in acquiring beginning basic-academic skills but more difficulty in acquiring advanced academic skills such as reading comprehension skills, math problem-solving skills, etc.

Reading comprehension can be problematic for children with ASD in that it requires a basic understanding of language [30], as well as executive function skills such as short-term working memory, decision making, and expressive language $[31,32]$. The relationship between reading comprehension and the language skills should be considered more in future investigations with both English and Non-English speaking children.

Our children with ASD, regardless of being HFA or LFA, did less well on writing skills, especially on writing their name, writing short sentences, or writing pinyin letters, when compared to age-matched peers. Writing appears to be an area of difficulty for our Chinese children with ASD regardless of their level of functioning.

In the area of math skills, both groups of Chinese children with ASD performed less well than did their control groups. However, the HFA group did perform better in math calculation, but less well on math wordproblems. The LFA group, in comparison, performed less well on both levels of math skills. These findings 
suggest that math might be an area of particular difficulty for Chinese children with ASD. These findings differ from Chiang and Lin [1] who reported that the majority of their students with HFA demonstrated average mathematical abilities. Although, in their study, the items that were included were primarily basic math calculations. Myles and Simpson [14], in another study, reported that their individuals with HFA had difficulty in math problem-solving tasks, similar to our current findings. Together, our current study and those of previous investigations suggest that individuals with ASD do not present difficulty in acquiring basic math skills, but present more difficulties in acquiring higher math skills like math-problem solving skills.

In conclusion, the current investigation suggests that despite poorer performances in several academic areas, our Chinese children with ASD acquire, at least, several basic academic skills. Additionally, many of our students even acquired high reading, writing, and math skills. This is a very encouraging finding that supports the need for educators, parents, and professionals to include these basic academic skills in their early treatment of children with ASD. However, more research is needed in this area to validate our findings.

\section{Comparison of ASD Groups}

When our two groups of Chinese children with ASD were compared together, we found that the HFA group performed much better overall in all the academic areas than did the LFA group. Yet, regardless of being high- or low-functioning, both groups of Chinese children performed remarkably alike in strengths and weaknesses across academic skills. For instance, in the reading area, both groups performed poorly in Print Awareness. Print Awareness (i.e., sometimes referred to as emergent literacy) and oral language are often related skills in most children [33-35], and especially in children with ASD [36]. That is, a strong relationship exists between language and emergent literacy in typically developing children (e.g., National Institute of Child and Health and Human Development [34], as well as in children with ASD [36]; thus, the problem with Print Awareness may be related to poor language skills in both of our groups of Chinese children with ASD. This implies that emergent literacy and language skills should be an early educational goal for Chinese children with ASD.

In addition, both groups of children with ASD performed well on basic math skills like counting and number identification; however, higher math skills were more difficult such as adding, subtracting, counting by $2 \mathrm{~s}, 3 \mathrm{~s}$, and $4 \mathrm{~s}$, and math problem solving skills. The HFA group was more successful than the LFA group. Thus, these findings indicate that math skills are an area of need in early intervention strategies for at least kindergarten age children who are HFA and LFA.

Finally, both groups of children with ASD had difficulty in the area of writing. This was especially true in writing sentences and paragraphs. It is possible that difficulties in writing skills could be related to our students learning to write pinyin rather than alphabet formation. Pinyin is an iconic form of writing and may require a higher skill level than learning to write phonetic-alphabet words. More research is definitely needed in this area for better clarification.

\section{FURTHER RESEARCH AND CONCLUSIONS}

The present study indicates that early academic skills can be acquired in both HFA and LFA Chinese children. Higher academic skills such as reading comprehension, writing, and advanced math are not as well developed. The current findings indicate Chinese children with HFA perform better on sight word reading and less well on reading comprehension, which parallels previous investigations. Print Awareness was found to be more problematic for both of our groups of Chinese children with ASD. It was postulated that difficulties in Print Awareness might be related to language issues in both groups. This investigation highlights the reading, writing, and math skills in young children with ASD, supporting the belief that academic learning is important and achievable. Early intervention in literacy, including reading, writing, and math skills, are obtainable goals and should be encouraged for young individuals with ASD, regardless of their level of functioning or disability. Additional research is needed with broader assessment in Chinese children with HFA and LFA.

\section{LEARNING OUTCOMES}

Readers will be able to describe the reading, writing, and math abilities among young Chinese children with ASD compared to Chinese children with Intellectual Disabilities or typical development.

\section{ABBREVIATIONS}

ASD = autism spectrum disorders

ID = intellectual disabilities 
TD $=$ typical development

HFA

$$
\begin{aligned}
= & \text { High functioning } \\
& \text { disorders }
\end{aligned}
$$

LFA

= Low functioning disorders

CADS $=$ Chinese Autism Diagnostic Scale

PDD-NOS $=$ Pervasive developmental disorder-Not otherwise specified

\section{REFERENCES}

[1] Chiang $\mathrm{H}$, Lin $\mathrm{Y}$. Mathematical ability of students with Asperger syndrome and high-functioning autism. Autism 2007; 11(6): 547-556.

https://doi.org/10.1177/1362361307083259

[2] Hao G, Layton TL, Zou X, Li D. Evaluating autism in a Chinese population: the Chinese Autism Diagnostic Scale. World Journal of Pediatrics 2014; 10(2): 160-163. https://doi.org/10.1007/s12519-014-0466-0

[3] Jones CRG, Happé $\mathrm{F}$, Golden $\mathrm{H}$, et al. Reading and arithmetic in adolescents with autism spectrum disorders: Peaks and dips. Neuropsychology 2009; 23(6): 718-728. https://doi.org/10.1037/a0016360

[4] American Psychiatric Association. Diagnostic and Statistical Manual-V (DSM-V) Development; 2013. Available at http: //www.psych.org

[5] Frith U, Snowling M. Reading for meaning and reading for sound in autistic and dyslexic children. British Journal of Developmental Psychology 1983; 1(4): 329-342.

https://doi.org/10.1111/j.2044-835X.1983.tb00906.x

[6] Minshew N, Goldstein G, Taylor HG, Siegel DJ. Academic achievement in high functioning autistic individuals. Journal of Clinical and Experimental Neuropsychology 1994; 16(2): 261-270.

https://doi.org/10.1080/01688639408402637

[7] Nation K, Clarke P, Wright B, Williams C. Patterns of reading ability in children with autism spectrum disorder. Journal of Autism and Developmental Disorders 2006; 36(7): 911-919. https://doi.org/10.1007/s10803-006-0130-1

[8] O'Connor N, Klein PD. Exploration of strategies for facilitating the reading comprehension of high-functioning students with autism spectrum disorders. Journal of Autism and Developmental Disorders 2004; 34(2): 115-127. https://doi.org/10.1023/B:JADD.0000022603.44077.6b

[9] Troyb E, Orinstein A, Tyson K, Helt M, et al. Academic abilities in children and adolescents with a history of autism spectrum disorders who have achieved optimal outcomes. Autism 2014; 18(3): 233-243.

https://doi.org/10.1177/1362361312473519

[10] Weitlauf A, Gotham K, Vehorn A, Warren Z. Brief Report: DSM-5 "Levels of Support:" A comment on discrepant conceptualizations of severity in ASD. Journal of Autism and Developmental Disorders 2104; 44(3): 471-476.

[11] Perfetti C, Landi N, Oakhill J. The acquisition of reading comprehension. In Snowling MJ, Hulme C, editors. The science of reading. Oxford: Blackwell Publishing 2005.

[12] Cronin K. The relationship among oral language, decoding skills, and reading comprehension in children with autism. Exceptionality 2014; 22(3): 141-157. https://doi.org/10.1080/09362835.2013.865531

[13] Ricketts J, Jones CRG, Happé F, Charman T. Reading comprehension in autism spectrum disorders: The role of oral language and social functioning. Journal of Autism and Developmental Disorders 2013; 43(4): 807-816.

https://doi.org/10.1007/s10803-012-1619-4

Myles BS, Simpson RL. Asperger Syndrome: A guide for educators and parents second edition. Austin, TX: Pro-Ed 2003.

[15] Estes A, Rivera V, Bryan, Cali P, Dawson G. Discrepancies between academic achievement and intellectual ability in higher-functioning school-aged children with autism spectrum disorder. Journal of Autism and Developmental Disorders 2011; 41(8): 1044-1052. https://doi.org/10.1007/s10803-010-1127-3

[16] Bae YS, Chiang H, Hickson L. Mathematical word problem solving ability of children with autism spectrum disorder and their typically developing peers. Journal of Autism and Developmental Disorders 2015; 45(7): 2200-2208. https://doi.org/10.1007/s10803-015-2387-8

[17] Mayes SD, Calhoun SL. Frequency of reading, math, and writing disabilities in children with clinical disorders. Learning and Individual Differences 2006; 16(2): 145-157. https://doi.org/10.1016/j.lindif.2005.07.004

[18] Griswold DE, Barnhill GP, Myles BS, Hagiwara T, Simpson $\mathrm{RL}$. Asperger syndrome and academic achievement. Focus on Autism and Other Developmental Disabilities 2002; 17(2): 94-102. https://doi.org/10.1177/10883576020170020401

[19] Myles BS, Barnhill GP, Hagiwara T, Griswold DE, Simpson $\mathrm{RL}$. A synthesis of studies on the intellectual, academic, social, emotional and sensory characteristics of children and youth with Asperger syndrome. Education and Training in Mental Retardation and Developmental Disabilities 2001; 36: 304-311.

[20] Schaefer Whitby P, Mancil GR. Academic achievement profiles of children with high functioning autism and Asperger syndrome: A review of the literature. Education and Training in Developmental Disabilities 2009; 44(4): 551-560.

[21] APA. Diagnostic and Statistical Manual of Mental Disorders IV. Arlington, VA: American Psychiatric Association 2000.

[22] World Health Organization. The ICD-10 Classification of Mental and Behavioral Disorders. Geneva: World Health Organization 1993.

[23] Chinese Society of Psychiatry. Chinese Classification of Mental Disorders (CCMD-3). Ginan, China 2005.

[24] Wechsler D. Wechsler Intelligence Scale for Children fourth edition. Hong Kong: King-May Psychological Assessment LTD 2010.

[25] Lecouteur A, Rutter Lord C. Autism Diagnostic InterviewRevised (ADI-R). San Antonio, TX: Pearson Publishing 2003.

[26] Constantine J. Social Responsive Scale. San Diego, CA: Western Psychological Services 2014.

[27] SPSS for Windows, rel 12. Chicago, IL: SPSS, Inc 2012.

[28] Kaiser $\mathrm{H}$. The application of electronic computers to factor analysis. Educational and Psychological Measurement 1960; 20: $141-151$. https://doi.org/10.1177/001316446002000116

[29] Stevens J. Applied multivariate statistics for the social sciences fourth ed.. Hillsdale, NJ: Erlbaum 2002.

[30] Antonacci P, O'Callaghan C. Promoting Literacy Development. Thousand Oaks, CA: Sage Publications 2012.

[31] Kibby M, Marks W, Morgan S, Long C. Specific impairment in developmental reading disabilities: A working memory approach. Journal of Learning Disabilities 2014; 37(4): 349363. https://doi.org/10.1177/00222194040370040601

[32] Stone C, Michaels D. Problem solving skills in learning disabled children. In Ceci, SJ editor. Handbook of cognitive, 
social and neuropsychological aspects of learning disabilities. Volume 2,( pp 291-315). Hillsdale, NJ: Erlbaum 1986.

[33] Gilliam R, Johnston J. Development of Print Awareness in language-disorder preschoolers. Journal of Speech, Language, and Hearing Research 1985; 28(10): 521-526. https://doi.org/10.1044/jshr.2804.521

[34] National Institute of Child Health and Human Development Early Child Care Research Network. Pathways to reading: The role of oral language in the transition to reading. Developmental Psychology 2005; 41: 428-442. https://doi.org/10.1037/0012-1649.41.2.428
[35] National Institute for Literacy. Developing Early Literacy: Report of the National Early Literacy Panel: A scientific synthesis of early literacy development and implications for intervention. Jessup, MD; 2008. Available from: www.nifl.gov]

[36] Lanter E, Watson L, Erickson K, Freeman D. Emergent literacy in children with autism: An exploration of developmental and contextual dynamic processes. Language, Speech, Hearing Services in Schools 2012; 43(3): 308-324.

https://doi.org/10.1044/0161-1461(2012/10-0083)

Received on 22-03-2017

Accepted on 31-03-2017

Published on 12-04-2017

DOI: https://doi.org/10.6000/2292-2598.2017.05.01.2

(c) 2017 Layton and Hao; Licensee Lifescience Global.

This is an open access article licensed under the terms of the Creative Commons Attribution Non-Commercial License (http://creativecommons.org/licenses/by-nc/3.0/) which permits unrestricted, non-commercial use, distribution and reproduction in any medium, provided the work is properly cited. 